\title{
Dynamic Webpage Design Based on CSS
}

\author{
TaoKuang ${ }^{1}$, Shanhong Zhu ${ }^{1,2}$ \\ ${ }^{1}$ School of Computer and Information Engineering, Xinxiang University, Henan, China \\ ${ }^{2}$ International School of Software, Wuhan University, Wuhan, China \\ 14878670@qq.com, kuangtaoxxu@163.com
}

\begin{abstract}
The traditional webpage using nested tables for webpage, this method has the advantages of simple operation, but generates redundancy HTML code which is not conducive to find and manage, prolix. however, CSS webpage layout is a concise code, more functional, higher flexibility of webpage layout design method. This article presents a method of personal blog of webpage design and application based on CSS.
\end{abstract}

Index Terms - table layouts, CSS, webpage design

\section{Introduction}

In recent years, personal blog is a kind of network diary's personal website form, it has neat interface, easy management, clear content and other characteristics, form (TABLE) layout and CSS layout is the construction of personal blog of two common web page layout method. Form has advantages of simple use, [1] fast production, and convenient manufaction etc, but its page code redundancy, structure and performance mixed together, CSS layout method makes up the deficiency of the layout of the form, separates performance and content, greatly simplify the code, reduce the network bandwidth resource waste, support the browser backward compatibility.

\section{Structural Layout}

CSS Cascading Style Sheets is the abbreviation, named "Cascading Style form". [2]CSS is the Web standard layout language, it can be effective for page layout, fonts, colors, backgrounds and other effects to achieve precise control. CSS has strong applicability, it can control the page and the appearance of the site, CSS can make the webpage more concise. The basic idea is: use DIV+CSS DIV to define the semantic structure; In whole on the < div > mark block, then to each block the CSS positioning, and eventually with CSS to beautify the web page, such as background, line frame, alignment attribute, etc. In each of the last add content, such as text, pictures, etc

With a personal blog as an example, using DIV+CSS layout to reconstruct this page, the page is divided roughly for top portion, the navigation section, the side, as in Figure 1 , The code is as follows:

$<$ body $>$

$<$ div id="header" $></$ div $>/ *$ head $* /$

$<$ div id="menu" $></$ div $>/ *$ menu*/

$<$ div id="main" $>/ *$ twi sub blocks in main block*/

$<$ div id="sidebar" $></$ div $>/ *$ sidebar*/

$<$ div id="content" $></$ div $>/ *$ content $* /$

$</$ div $>$

$<$ div id="footer" $></$ div $>/ *$ foot $* /$

$<$ /body $>$
Among them, in order to realize the webpage display center,[3] set the box margin property right is 0 auto, in order to achieve the middle two column display, set the left float properties to left, main float properties to right, in order to achieve the border effection, set the border property to 1 frames, as shown in Figure 2.

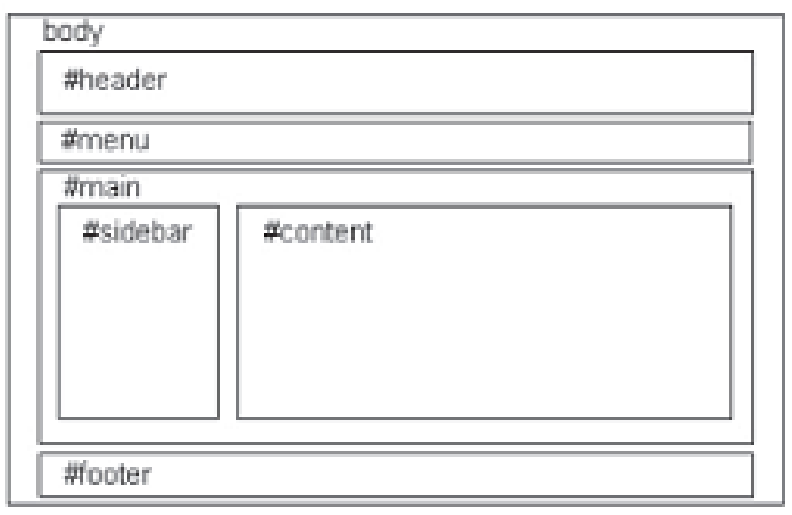

Fig. 1 page structure

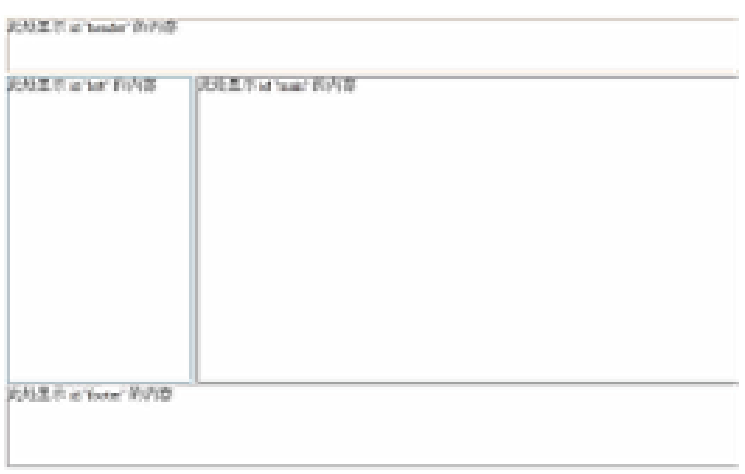

Fig 2. Page effect

\section{Navigation List Display}

Link dynamic display of webpage design is a very important technology, [4]there are 4 kinds of links to state the most widely used, No access link is a: link, visited links is a: visited, hovering the mouse pointer in the link state is a: hover, being activated to link is a: active, in webpage design, through the definition of link, according to the link visited hover active order defined below , The following settings link at the top region style, add a navigation bar. as shown in Figure 3. 


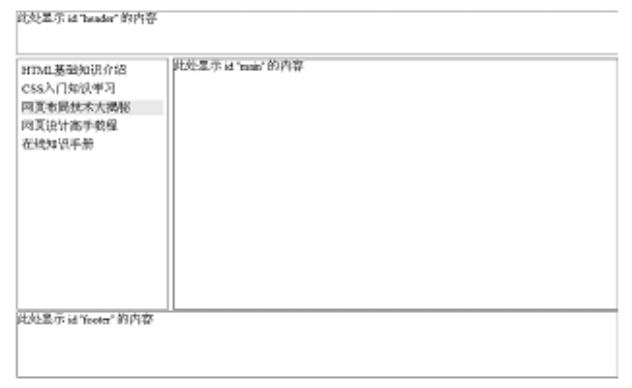

Fig .3 Link Style

\section{Graphic Mixed Style}

Mixed graphic and text content is the important content in the webpage layout, [5]only by mixed, the boring text can become vivid, colorful pictures and text orderly arranged together, The following is the implementation of mixed graphic and text effect in \#main block, as shown in Figure 4,codes as follows:

$<$ h2 $>$ CSS webpage layout technology application research $\langle/ \mathrm{h} 2\rangle$

$<$ img src=" /study.jpg" width=360 height=280/>

$<\mathrm{p}>$ The use of Table page layout is the traditional webpage layout display technology, along with the Web2standard design concept of universal $</ p>$

$</$ div $>$

CSS style settings are as follows

\#mainh2 \{text-align:center;color:\#F00;margintop: $10 \mathrm{px} ;\}$

\#main img \{float:left;height:220px;margin:15px;

\#mainp \{ margin-top:15px;

padding:2px;font-size:14px;text-

indent:2em;color:\#03F;

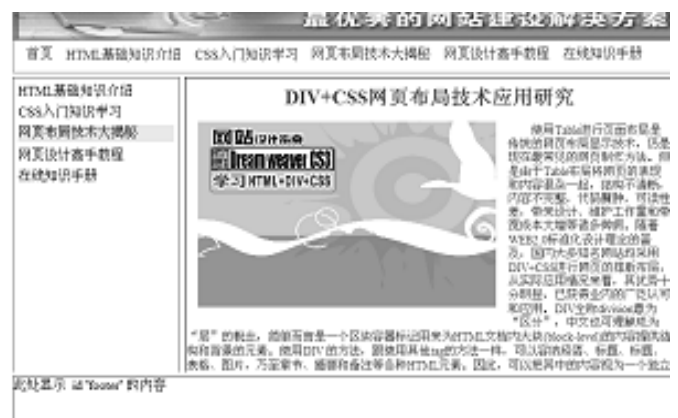

Fig 4. Mixed Style

\section{Conclusions}

The CSS standard designing blog website has the following advantages: due to the content of performance and structure are completely separated, website construction of the division of personnel become more clear, development and maintenance efficiency is obviously improved, correcting also become easier, such as with a page, the same content, and the use of different CSS files can realize different real time blog update effect, high degree of streamlining the code page, page loading speed, not only saves a lot of bandwidth, but also can improve search engine search procedure efficiency, webpage content more easily indexed by search engines, brings the user better experience.

\section{References}

[1] Jones, D.Navin-Chandra, D. IndustryNet: a model for commerce on the World Wide Web, IEEE EXPERT, 1990.

[2] O'Connor, G., O'Keefe, B. Viewing the Web as a Marketplace: The Case of Small Companies, forthcoming in Decision Support Systems, 1997

[3] Benjamin, R., Wigand, R.T. "Electronic Markets and Virtual Value Chains on the Information Highway," Sloan Management Review, 1995,pp 62-72.

[4] Martin Libicki, James Schneider, Dave R. Frelinger, and Ann Slomovic. Scaffolding the New Web: Standards and Standards Policy for the Digital Economic. RAND, Santa Monica, CA, 2000.

[5] Jones, D.Navin-Chandra, D. IndustryNet: a model for commerce on the World Wide Web, IEEE EXPERT, 1990.

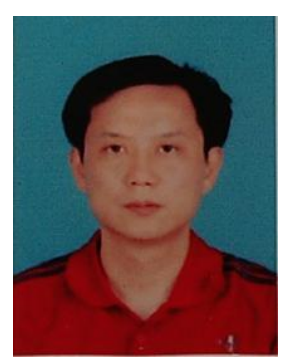

Tao Kuang, was born in China in 1975. He earned a master's degree in software engineering in the School of Information Engineering at Zhengzhou University. $\mathrm{He}$ is employed as a Associate Professor in Xinxiang College, where he is now a director of the Department of Network and Information Security Department. His research interests include computer multimedia, virtual reality application, embedded system and application, data mining, computer communication and so on. 